7. Ю.П. Кузнецов, В.Л. Химич, С.Н. Хрунков, А.Б. Чуваков, Р.А. Погодин. Исследование характеристик радиальной реактивной турбины // Известия высших учебных заведений. Авиационная техника. 2020. №4.

8. Кузнецов Ю.П., Чуваков А.Б. Многовенечная радиальная турбина // Патент на полезную модель RU 110132, U1. Опубл. 10.11.2011, бюл. № 31

9. Кузнецов Ю.П., Чуваков, А.Б., Погодин Р.А. Турбомашина // Патент на полезную модель RU 193118, U1. Опубл. 15.10.2019, бюл. № 29

\title{
Кузьмин М.В. \\ Теоретические основы и методика аналитического прогнозирования (на основе математической теории отношений толерантности)
}

Российский государственный аграрный заочный университет (Россия, Балашиха)

doi: $10.18411 / \mathrm{sr}-10-08-2021-18$

\section{Аннотация}

На основе математической теории отношений толерантности разработан метод прогнозирования форм рабочих органов машин до разработки законченных технических решений (на допатентном уровне), позволяющий выявить их преимущества и доказательно констатировать их работоспособность. Разработана методика прогнозирования. Прогнозирование может проводиться тремя методами: применением теории графов, использованием прототипов объектов, использованием теоремы Кальмара - Якубович. Методами аналитического прогнозирования определены перспективные рабочие органы, в том числе с.-х. машин. Экспериментально доказано, что повышение удельной производительности у решетной очистки составляет 2,5 - 3 раза, у триера 5 и более раз.

Ключевые слова: прогнозирование, толерантность, математическая теория отношений, рабочий орган, теория, прототип, методика, удельная производительность.

\section{Abstract}

Based on the mathematical theory of tolerance relations, a method has been developed for predicting the shapes of working bodies of machines before developing legal technical solutions (at the pre-patent level), which allows to reveal their advantages and to establish their operability with evidence. A forecasting technique has been developed. Forecasting can be carried out by three methods: using graph theory, using prototypes of objects, using the Kalmar-Yakubovich theorem. The methods of analytical forecasting have identified promising working bodies, including agricultural machines. It is experimentally proved that the increase in the specific productivity of sieve cleaning is $2.5-3$ times, for trier 5 or more times.

Keywords: forecasting, tolerance, mathematical theory of relations, working body, theory, prototype, methodology, specific productivity.

Теоретические основы прогнозирования. Для ускорения разработки принципиально новых способов и устройств разработан метод прогнозирования, который позволяет определять общие формы рабочих органов машин до разработки законченных технических решений (на допатентном уровне), позволяющий выявить их преимущества и доказательно констатировать их работоспособность.

Поставить на научную основу решение этой задачи позволяет наличие основополагающих (фундаментальных) общностей в формах рабочих органов, являющихся (как установил В.П. Горячкин) развитием пространственного трехгранного клина [1]. С учетом этого даже принципиально новые рабочие органы находятся с известными в определенных отношениях, поэтому для решения указанной 
задачи нами разработан и использован метод прогнозирования на основе математической теории отношений толерантности.

Толерантность (от лат. Tolero - содержать в себе) есть аксиоматически строгое определение сходства. Толерантность как разновидность отношений является переносом понятий сходства, неразличимости, одинаковости в область строгой математической теории. Условие толерантности означает, что если X характеризуется признаками $\mathrm{x}_{1}, \mathrm{x}_{2}, \ldots, \mathrm{x}_{\mathrm{i}}, \ldots$, то есть $\mathrm{X}\left(\mathrm{x}_{1}, \mathrm{x}_{2}, \ldots, \mathrm{x}_{\mathrm{j}} \ldots\right)$, а $\mathrm{Y}\left(\mathrm{y}_{1}, \mathrm{y}_{2}, \ldots, \mathrm{y}_{\mathrm{i}}\right)$, то при $\mathrm{X} \wedge \mathrm{Y} \neq$ $\varnothing$ имеет место совпадение нескольких признаков, в крайнем случае, любого одного признака. Следовательно, отношение толерантности может быть задано набором признаков таким образом, что объекты с общими признаками (хотя бы одним) будут толерантными [2]. Это даёт возможность при определённых условиях переносить закономерности функционирования с апробированных объектов на толерантные им, но не апробированные объекты (новые рабочие органы) и тем самым доказательно констатировать их работоспособность. Пусть элементами множества М являются технические объекты $\mathrm{X}_{1}, \mathrm{X}_{2}, \ldots$, например рабочие органы сельскохозяйственных машин, объединённые определёнными общими признаками (хотя бы одним). Каждый рабочий орган $\mathrm{X}_{\mathrm{j}}$ характеризуется кортёжем $\mathrm{S}_{\mathrm{j}}$. Каждый кортёж состоит из набора элементов, характеризующих рабочий орган: функций $F_{i j}$, описывающих закономерности взаимодействия рабочего органа с обрабатываемой средой, уравнение движения и др., свойств или параметров рабочего органа $\mathrm{C}_{\mathrm{i}}$, Совокупность функций запишем в виде $\bigcup_{j=1}^{m} F_{i j}\left(X_{i}\right)$, соответственно совокупность свойств запишем как $\bigcup_{j=1}^{K} C_{i j}$, где оператор $\bigcup$ - знак совокупности, дизъюнкции без "или". С учетом этих обозначений кортёж, характеризующий рабочий орган, запишется в виде

$$
S \equiv\left\langle\bigcup_{j=1}^{m} F_{i j}, \bigcup_{j=1}^{K} C_{i j},\right\rangle
$$

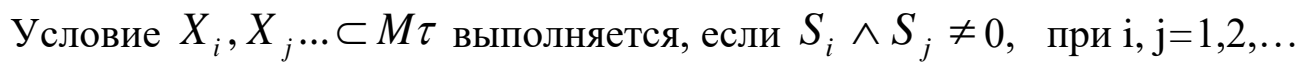

Отношение толерантности можно задать другим способом. Пусть операция $\varphi$ ставит в соответствие элементу $\mathrm{X}(\mathrm{X} \subset \mathrm{M})$ его образ $F(X)$ в множестве $L$; то есть имеет место соответствие $\varphi: M \rightarrow L$. Толерантность $A \varphi$ элементов $\mathrm{X}, \mathrm{y}$ на множестве м $X A \varphi Y$ задаётся условием $F(X) \wedge F(Y) \neq 0$. Отношение $A \varphi$ обладает следующими свойствами: симметричность $F(X) \wedge F(Y)=F(Y) \wedge F(X)$, рефлексивность, которая имеет место только тогда, когда $\varphi$ определено на всём множестве М, $F(X) \wedge F(Y) \neq 0$ [2]. Если для $X$ отношение $A \varphi$ не рефлексивно, то $F(X)=0$, и соотношение $X A \varphi Y$ не выполняется ни для какого $Y$, так как $F(X) \wedge F(Y)=0 \wedge F(Y)=0$.

Методика прогнозирования на основе математической теории отношений толерантности. Базой прогноза являются любые объективные и достоверные источники информации: патентные, литературные, отчеты по НИР и ОКР и др. Время основания прогноза и упреждающий период не ограничены.

При прогнозировании было использовано три метода представления пространства толерантности и его трансформации: применение теории графов, использование прототипов объектов и теоремы Кальмара - Якубович.

Основанное на этих положениях прогнозирование содержит следующие этапы: 
1. Выделение группы рабочих органов $X_{\mathrm{i}}$ на основе фундаментальных общностей $\tau$, образующих множество $\mathrm{M}\left(\mathrm{X}_{\mathrm{i}} \subset \mathrm{M}\right)$, то есть образование пространства толерантности М $\tau$, где $\tau$ - отношение толерантности.

2. Анализ параметров выделенных рабочих органов машин (свойств, закономерностей функционирования и ограничивающих факторов, препятствующих интенсификации) на основе источников информации, заложенных в базу прогноза.

3. Выделение из М $\tau$ анализом подпространств толерантности и нетолерантности форм рабочих органов, реализующих интенсификацию рабочего процесса и повышающих его оценочные показатели.

4. Верификация результатов. Она может быть выполнена любым применяемым в прогнозировании методом.

Любой объект $\mathrm{X}$ характеризуется набором параметров $\mathrm{x}_{\mathrm{i}}$ (свойств, закономерностей воздействия на обрабатываемый материал, показателями качества работы и др. $)$ - X $\left(\mathrm{x}_{1}, \mathrm{x}_{2}, \ldots, \mathrm{x}_{\mathrm{i}}, \mathrm{x}_{\mathrm{n}}\right)$, который обычно именуют кортёжем. Толерантность рабочих органов $\mathrm{X}$ и $\mathrm{У}(\mathrm{X} \tau \mathrm{Y})$ определяется условием, что пересечение их кортежей не пусто [2]:

$$
\left(\mathrm{x}_{1}, \ldots \mathrm{x}_{\mathrm{n}}\right) \wedge\left(\mathrm{y}_{1}, \ldots \mathrm{y}_{\mathrm{n}}\right) \neq 0 .
$$

Тогда параметры толерантных рабочих органов, входящие в пересечение (2), эквивалентны ( $\mathrm{x}_{\mathrm{i}} \equiv \mathrm{y}_{\mathrm{i}}, \mathrm{x}_{\mathrm{j}} \equiv \mathrm{y}_{\mathrm{j}}$ и т. д.) в пространстве толерантности $\mathrm{M} \tau$, где $\mathrm{X} \subset \mathrm{M}$, $\mathrm{У} \subset \mathrm{M}, \tau-$ признак толерантности. Условие (2) можно представить в виде

$$
\left(\Pi_{\mathrm{x}}, Э_{\mathrm{x}}\right) \wedge\left(Э_{\mathrm{y}}, \mathrm{H}_{\mathrm{y}}\right) \neq 0,
$$

где $\Pi_{\mathrm{x}}, Э_{\mathrm{x}}, Э_{\mathrm{y}}, \mathrm{H}_{\mathrm{y}}-$ подмножества функционально определенных закономерностей (характеристик) прототипа Х(П $\left(\bigcap_{\mathrm{x}}, Э_{\mathrm{x}}\right)$, и нового рабочего органа У( $\left.Э_{\mathrm{y}}, \mathrm{H}_{\mathrm{y}}\right)$;

$Э_{\mathrm{x}}, Э_{\mathrm{y}}$ - эквивалентные подмножества характеристик прототипа и нового рабочего органа $\left(Э_{\mathrm{x}} \equiv Э_{\mathrm{y}}\right)$, образующие пересечение;

— не входящие в пересечение (3) характеристики прототипа: $\left(\Pi_{\mathrm{x}}\right)$ - его недостатки, и нового рабочего органа $\left(\mathrm{H}_{\mathrm{y}}\right)$ - его преимущества.

При прогнозировании целесообразно выбрать такое отношение толерантности $\tau$, чтобы оно характеризовало фундаментальную общность форм рассматриваемых рабочих органов.

В связи с тем, что в результате прогнозирования определяются общие формы $\mathrm{PO}$, а не технические решения по определенным рабочим органам, их параметры в первом приближении можно принять аналогичными прототипам. Прогнозирование может проводиться тремя методами: применением теории графов, использованием прототипов объектов, использованием теоремы Кальмара - Якубович.

Первый метод (применение теории графов). При обозначении элементов множества $M$ натуральными числами $(\mathrm{I}, 2,3, \ldots \mathrm{p})$ получим совокупность подмножества $S_{p}$ числового ряда. Толерантность элементов $X$ и $Y$, характеризуемых подмножествами, входящими в указанный числовой ряд, определяется условием $S_{p i} \wedge S_{p j} \neq 0$. Графически элементы множества $S_{p}$ удобно представить вершинами графа. Тогда рёбра, соединяющие эти вершины, будут отображать соответствующие отношения толерантности между элементом, находящимся на ребре, инцидентном вершинам, и элементами, расположенными в его вершинах. Элементы, находящиеся на ребре, будут иметь кортёж, являющийся объединением кортежей элементов, находящихся в вершинах графа, которые это ребро соединяет. При расположении в вершинах графа известных технических объектов $X_{1}, X_{2}, \ldots$ можно прогнозировать новые технические объекты $X_{12}, X_{23}, \ldots$, расположенные на рёбрах графа и толерантные $X_{1}, X_{2}, X_{3}, \ldots$ Они будут объединять свойства элементов $X_{1}, X_{2}, X_{3} \ldots:\left(X_{12}, X_{23}, X_{34}, \ldots\right)$ с таковым, размещённым на смежных вершинах и обладающие совокупностью их свойств. При этом необходимо рассматривать подмножества характеристик прототипов объектов $\mathrm{m}$ 
$(1)$

m (2), m (3). Их объединение будет характеризовать свойства объектов m $(1,2), \mathrm{m}(2,3)$, $\mathrm{m}(1,3)$. При этом подмножества характеристик $\mathrm{H}_{\mathrm{y}}$, не входящих в пересечение (3), определяют свойства новых (гибридных) рабочих органов. Первым методом можно получать объекты, объединяющие свойства двух и более объектов.

Второй метод (использование прототипов объектов). При прогнозировании с использованием прототипов объектов, преобразованием которых могут быть определены объекты с желательными признаками, необходимо рассмотреть подмножества $\Pi_{x}, \mathrm{H}_{y}$, не входящие в пересечение (3). Характеристики, содержащие недостатки прототипа $\left(\Pi_{\mathrm{x}}\right)$ должны быть исключены таким изменением схемы прототипа, при которой новый рабочий орган приобретёт преимущества, определяемые одмножеством $\left(\mathrm{H}_{\mathrm{y}}\right)$. Содержание изменения является творческим процессом. Второй метод - задание отношения толерантности на основе прототипов $X_{1}, X_{2}, \ldots \subset \mathrm{M}$ - даёт возможность переносить закономерности функционирования с апробированных рабочих органов на толерантные им не апробированные рабочие органы (новые рабочие органы) и тем самым доказательно констатировать их работоспособность.

Третий метод (использование теоремы Кальмара - Якубович). Если соответствие $\varphi: M \rightarrow L$ задается функциональной зависимостью - образ $X$ в $L$ есть однозначная функция $F(X)$, то отношение $A \varphi$ транзитивно, так как при $X A \varphi Y$ и $Y A \varphi Z \quad$ соответственно определяет $F(X)=F(Y) \quad$ и $\quad F(Y)=F(Z)$, то $F(X)=F(Z)$, и имеет место соотношение $X A \varphi Z$. Следовательно, всюду определенное соответствие $\varphi: M \rightarrow L$, порождает во множестве $M$ симметричное и рефлексивное соответствие - отношение толерантности. Это заключение составляет содержание теоремы Кальмара - Якубович [2]: «Произвольное отношение толерантности на множестве $M$ можно задать как отношение $A \varphi$ с помощью некоторого всюду определенного соответствия $\varphi: M \rightarrow L »$.

При прогнозировании с использованием теоремы Кальмара - Якубович на

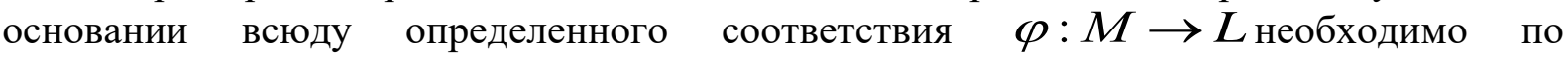
закономерностям, действующим в $L$, определить формы толерантных рабочих органов в множестве $M$. Затем преобразованием этих рабочих органов получить их новые формы, на которые не действуют ограничивающие факторы. При прогнозировании этим методом желательно использовать материалы метатеории сельскохозяйственных машин, но в связи с её недостаточным развитием следует использовать материал теории сельскохозяйственных машин. Таким образом, третий метод даёт возможность на основе закономерностей, действующих на известные рабочие органы, определять толерантные им формы новых рабочих органов.

Выводы. Изложенный метод прогнозирование имеет существенные преимущества по сравнению с известными. Он даёт возможность методами прогнозирования определять перспективные формы рабочих органов технических систем до разработки их теории, до оформления в виде законченного технического решения, проведения экспериментальных исследований и доказательно констатировать их работоспособность и эффективность.

Основанием прогноза могут служить самые разнообразные источники информации, а также работающие технические системы. Для обоснованности прогноза достаточно единичных источников информации или образцов, поэтому нет необходимости в статистической выборке материала большого объема. 
Перевод научно-технической информации в прогнозную проводится на основе аналитических зависимостей и свойств известных технических систем без привнесения субъективных факторов. Поэтому данный метод определён как аналитическое прогнозирование.

Впервые при прогнозировании нет необходимости в преобразовании качественных признаков в количественные оценки.

Так как общие формы не подлежат защите охранными документами, то полученные результаты прогнозирования могут использоваться другими лицами и организациями для создания технических решений с последующей защитой созданной ими интеллектуальной собственности.

Методами аналитического прогнозирования нами определены два вида форм перспективных рабочих органов: безвальные спирально-винтовые (БСВ) и гибкие в виде тел вращения с участком обратной кривизны. Результаты прогнозирования с изображением схем рабочих органов предполагается опубликовать в следующем номере журнала.

БСВ сепарирующие рабочие органы при экспериментальной проверке показали повышение удельной производительности в $2,5-3$ раза (решета) и в 5 и более раз (триеры) [3].

1. Горячкин В.П. Собрание сочинений. В 3 т. Т 1 [Текст] / В.П. Го-рячкин. - М.: Колос, 1965. - 720 с.

2. Шрейдер Ю.А. Равенство, сходство, порядок [Текст] / Ю.А. Шрей-дер. - М.: Наука, 1971. - 254c.

3. Кузьмин М.В. Нетрадиционные рабочие органы для технико-технологической модернизации сельскохозяйственного производства: дис... д-ра техн. наук: 11. 11.2009 / М.В. Кузьмин. - М.: РГАЗУ, 2009. - $430 \mathrm{c.}$

\section{Ткаченко С.С., Емельянов В.О., Мартынов К.В. Пористое кварцевое стекло, как связующее в литейной керамике} Филиал РАХ "Творческая мастерская "Литейный двор" doi: 10.18411/sr-10-08-2021-19

(Россия, Санкт-Петербург)

\section{Аннотация}

В статье приведены экспериментальные данные о влиянии водной дисперсии акрила на структуру кремнегеля. Представлены исследования микроструктуры кремнегеля методом электронной микроскопии. Показано действие водной дисперсии акрила на формирование структуры связующего, как фрактального объекта. Установлено комплексное действие органических полимеров на систему золь-гель. Сделан вывод о возможности использования водной дисперсии акрила для формирования заданной структуры геля кремнекислоты.

Ключевые слова: структура, прочность, гель, структура, кремнезоль, модификатор.

\section{Abstract}

The article presents experimental data on the effect of acryl (ACR) water dispersion on silica gel structure. Studies of silica gel microstructure by electron microscopy are presented. The effect of ACR water dispersion on the binder structure formation as a fractal object is shown. The complex effect of organic polymers on the sol-gel system was found. The conclusion is made about the possibility of using ACR water dispersion to form a given structure of a silica gel.

Keywords: structure, strength, gel, structure, siliceous, modifier. 